\title{
Study of Cataclysmic Variables with LDS Sky Surveys and ESA Gaia
}

\author{
René Hudec*广 \\ Czech Technical University in Prague, Faculty of Electrical Engineering, Prague, Czech \\ Republic, and Kazan Federal University, Russian Federation \\ E-mail: rene.hudec@gmail.com \\ Vojtěch Šimon \\ Czech Technical University in Prague, Faculty of Electrical Engineering, Prague, Czech \\ Republic, and Astronomical Institute, AS CR Ondrejov, Czech Republic \\ E-mail: vojtech.simon@gmail.com
}

\begin{abstract}
The goal of this paper is to discuss the capabilities of ESA satellite missions Gaia and of the photographic LDS surveys for investigation of cataclysmic variables (CVs). We show that both of them can contribute to the study of CVs and related objects. Spectrophotometry and lowdispersion spectroscopy are the most important for CV analyses with the observations of Gaia. We present the possible strategies for investigation of CVs in the sampled photometric and spectroscopic data provided by Gaia. E.g. statistical properties of the long-term activity of various types of $\mathrm{CVs}$ can be determined from them.
\end{abstract}

The Golden Age of Cataclysmic Variables and Related Objects - III, Golden2015

7-12 September 2015

Palermo, Italy

\footnotetext{
* Speaker.

${ }^{\dagger}$ I acknowledge grant 13-33324S by GACR
} 


\section{Introduction}

Although the two projects covered by this contribution, namely Gaia and LDS photographic surveys, are based on different image recording techniques, and cover different epochs, both of them can effectively contribute to the investigation of cataclysmic variables (CVs).

\section{The ESA Gaia mission}

The ESA satellite Gaia was successfully launched on December 19, 2013. Gaia is an ambitious mission to chart a three-dimensional map of our Galaxy, in the process revealing the composition, formation and evolution of the Galaxy (e.g. Eyers et al. 2013). Gaia will provide unprecedented positional and radial velocity measurements with the accuracies needed to produce a stereoscopic and kinematic census of about one billion stars in our Galaxy and throughout the Local Group. This amounts to about 1 percent of the Galactic stellar population.

\subsection{Astrophysics with Gaia}

Motivation of this paper is to outline the possibilities of performing astrophysics of CVs with Gaia data. This is not trivial as the main goal of the Gaia mission is to create a catalog. The photometric sampling provided by Gaia will not be optimal for many variable astrophysical sources. However, the fine spectrophotometry (in reality ultra-low-resolution spectroscopy) provided by BP/RP photometers will be unique and important for many astrophysical investigations with Gaia, including cataclysmic variables science (Hudec \& Šimon 2007a and 2007b; Hudec et al. 2012 and 2013). Analogous investigations can be performed with photographic low dispersive spectroscopy (LDS) surveys, which cover many decades and allows the long-term variations to be studied.

In addition to that, for some important CVs features such as outbursts and/or high/low state transitions (characteristic timescales or quaiperiods) even the specific Gaia sampling can provide valuable photometric data. We also note that even astrometric parameters derived by Gaia may be useful as the parallaxes may determine distances and absolute magnitudes of numerous CVs.

Even the sampled Gaia data can provide the following properties of the object:

1. Determination of amplitude of the brightness variations

2. Statistical distribution of brightness

3. Absolute magnitude (from distance determined from parallax (and interstellar extinction)). Note that these items 1,2, and 3 even enable to establish the physically justified sequence of types of CVs.

4. Color indices and their time variations

In principle, it is also possible to search for the periods and cycles of the brightness variations, but one has to very cautious in doing this procedure using the sampled data from Gaia.

The sampling of the data provided by Gaia is not optimal for the astrophysical work; these observations are not dense enough and not equidistantly distributed. Typically, one can expect 50100 photometric points over 5 years. However, additional data can be provided by the ground-based experiments. 
We propose the following two approaches for classification and verification of CVs in the sampled Gaia data. The first one is to search for outbursts or high/low state transitions, i.e. analysis of parameters of the light curve not strongly dependent on sampling.

\subsection{Statistical properties of the long-term activity of CVs, perspectives of histograms for Gaia}

We investigated the profiles of the light curves of CVs of various types with the real observations from the AFOEV database (daily means). We approximated the Gaia sampling of these light curves by selecting the data separated by $\sim 20$ days. This procedure yielded the amount of the sampled data and the length of the mapped time segment which roughly corresponded to the expected time of observing with Gaia. We investigated which methods can yield the correct identification of a CV of a given type almost independent of the sampling. We identified the impact of the Gaia data on the investigation of CVs.

The basic profile of the high state and its fluctuations in e.g. novalike systems and supersoft X-ray binary sources can be plausibly mapped by the sampled Gaia data. Only the high state/low state transitions may be missed or covered by only one or two data points by Gaia. On the other hand, we expect the large outbursts of a dwarf nova to be covered by only a very few data points while short outbursts may be missed in the Gaia data set. Moreover, the individual outbursts will be probably captured in different phases due to their short duration. Gaia will therefore usually not provide us with information about the profile of the outburst. However, we found that the statistical distribution of magnitudes of a given $\mathrm{CV}$ is only slightly distorted if a long time (several years) interval is covered. The statistical distribution of brightness and its parameters like the standard deviation, skewness, excess are a representative description of the properties of the longterm activity of CVs. These histograms are only slightly distorted by the sampling of the Gaia observations if a long (several years) time segment is covered by the observations. Nevertheless, a distortion may appear if the recurrence time of outbursts is close to the sampling time of Gaia.

We conclude that the sequence of the types of CVs, justified by the physics of accretion onto the compact objects, is reflected in the statistical distribution of the long-term brightness variations, hence it is suitable for analysis of the sampled Gaia data. The importance of this sequence can be even enhanced if the apparent magnitudes are transformed to the absolute magnitudes by using the distances of CVs determined from the parallaxes.

\subsection{Rare flares (different from outbursts in dwarf novae) in CVs}

We investigated the possibility to detect and identify rare phenomena in CVs with Gaia. Deep monitoring of a large number of objects can lead to discoveries of more CVs with flares (phenomena different from outbursts) like the event discovered by van Amerongen \& van Paradijs (1989) and to detection of additional such events even in the already known systems. We investigated some CVs in the Bamberg photographic plates which have a similar sampling and coverage as the expected Gaia data. We conclude that the discovery of such events is possible with the monitoring planned for Gaia (Šimon 2010). 


\subsection{Ultra-low-dispersion spectra}

The ultra-low-dispersion spectra will be provided by Gaia RP/BP photometers. They can play a significant role in (1) searches for prominent spectroscopic variability, and (2) searches for objects with prominent (maybe variable) spectral features.

As an example, the spectra of the outburst of V407 Cyg (Munari et al. 2011) (or of an analogous object) could be investigated by Gaia. The spectral appearance of V407 Cyg in outburst was a highly peculiar one. The spectrum of this event is completely different from those ever recorded for this object and other symbiotic Mira variables. The white dwarf companion to the Mira variable experienced an outburst similar to that of classical novae, and its ejecta were moving in the circumstellar environment already filled by the ionized wind of the Mira.

We summarize several types of CVs and the related systems with very bright emission lines:

- Supersoft X-ray binary sources in the optical high states (Steiner \& Diaz 1998). Their spectra display intense and broad emission lines (especially H $\alpha$ ) (e.g. V Sge (Herbig et al. 1965)). Such systems also often have Balmer jump in emission.

- Classical novae in the nebular phase of the outburst (late phase, weeks to months after the maximum of the optical luminosity) - faint continuum, very strong emission lines of various elements (e.g. Munari et al. 2013).

- Symbiotic systems: very strong emission lines (mainly H $\alpha$ ) (e.g. Davidson et al. 1978).

- Dwarf novae: highly variable strong Balmer jump (e.g. Walker \& Chincarini 1968).

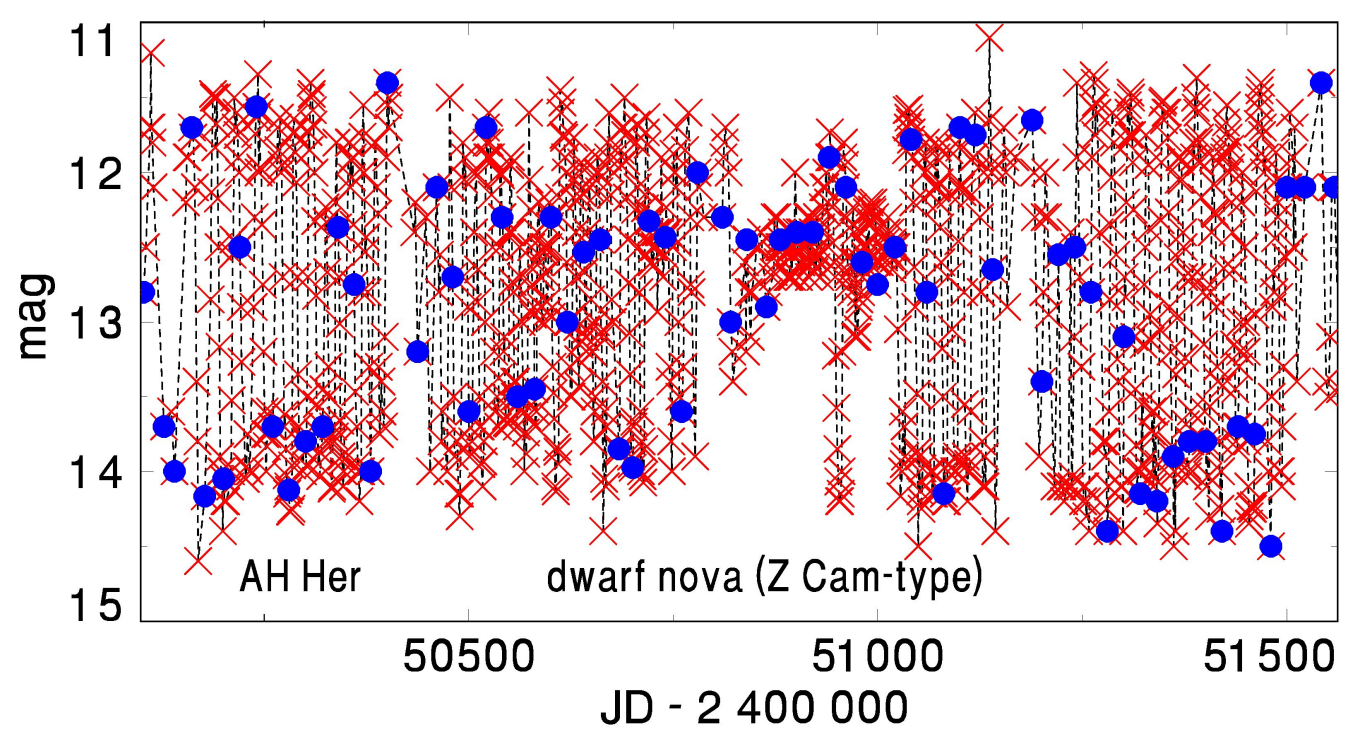

Figure 1: Comparison of the observed (one-day means of AFOEV data (crosses)) and the approximated sampled data of the dwarf nova AH Her (closed circles). Heavily sampled data are expected from the Gaia satellite. Notice that it is difficult to resolve the profile of the light curve in the sampled data. 


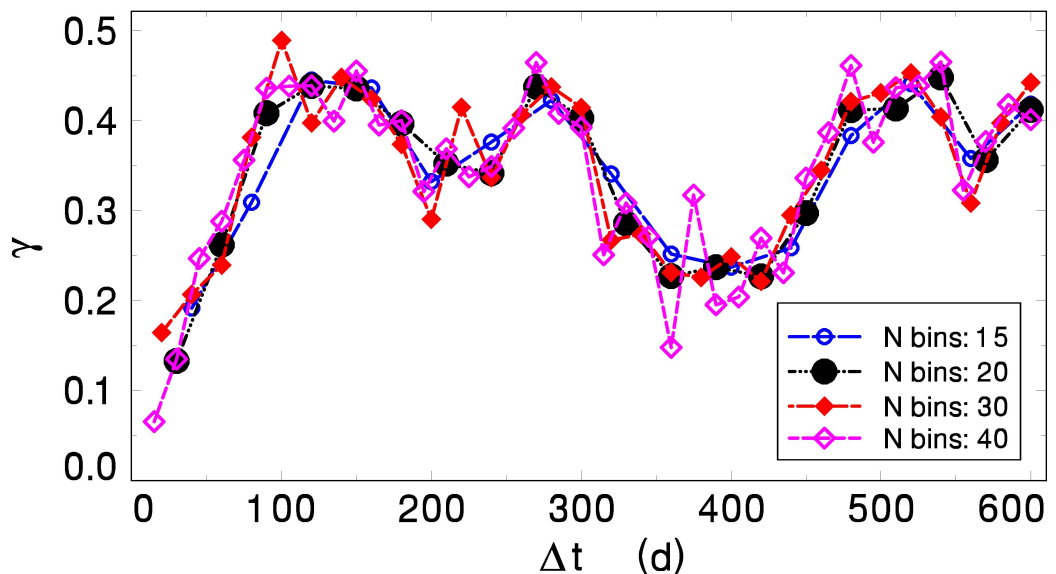

Figure 2: Variogram for the approximated Gaia data of the supersoft X-ray source V Sge. A higher value of $\mathrm{N}$ bins can yield a better determination of the possible cycle-length, but also a bigger noise. 


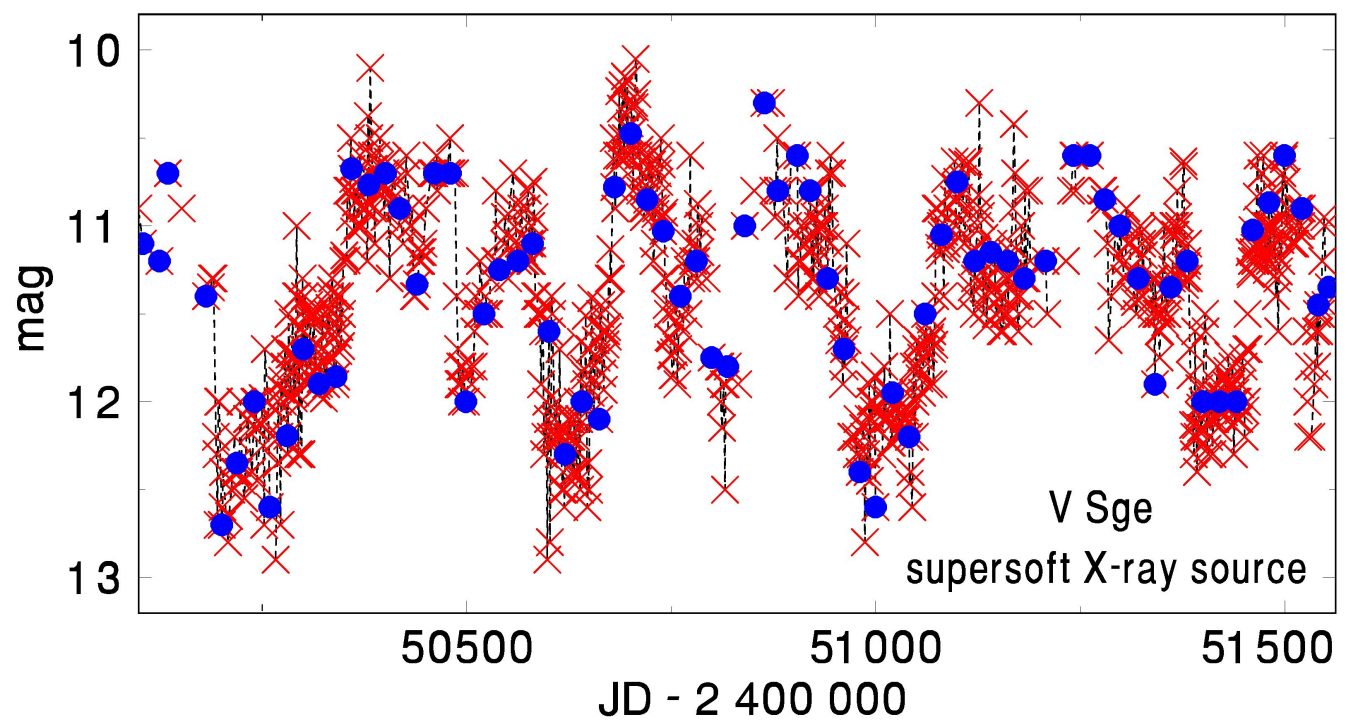

Figure 3: Comparison of the observed (one-day means of AFOEV data (crosses)) and the approximated sampled data (closed circles) of the supersoft X-ray source V Sge. Heavily sampled data are expected from the Gaia satellite.

\subsection{The color indices from RP/BP spectra}

The color indices can be determined from the spectra obtained by the Gaia RP/BP photometers. Significant results are expected to be immediately available, there is no need to wait for years as in the photometry case.

The color indices give important information on the spectral energy distribution. These indices can play an important role in classification and/or verification of CVs. They are also important for a search for the common properties of the sources of a given kind (e.g. to help identify a source as a $\mathrm{CV}$ and/or to study the evolution of the spectral profile with time as the CV undergoes various states of its activity). Not only the color indices of the object at a given time, but also the time evolution of these indices are therefore important. They can also play a role in resolving among the individual radiation mechanisms (e.g. cyclotron radiation versus thermal emission). Even variations of strong emission lines with respect to the continuum can be resolved by the color indices (e.g. $\mathrm{H} \alpha$ changes, changes of Balmer jump between emission and absorption). The color indices are also important for forming a representative ensemble of events (e.g. outbursts) in a given $\mathrm{CV}$ or in a given type of CVs. This is possible also for faint objects detected by Gaia (down to mag $\sim 20$ ).

The parallaxes from Gaia will enable to determine the distances, and hence the absolute magnitudes of many CVs. It will be therefore possible to determine and study the relation between the color indices and the absolute magnitudes of $\mathrm{CVs}$ of various types.

\subsection{Searches for cycles or periods}

Variograms allow us to search for the characteristic timescales or quasiperiods which extend just for several epochs of the cycle. Variogram characterizes the spatial continuity or roughness of a data set. Variograms are important for a search for the superorbital cycles in the long-term activity 
of CVs and low-mass X-ray binaries because their long-term activity is not periodic. Standard period searches therefore often reveal nothing.

We used the light curves of CVs of various types for testing. We applied the real observations from the AFOEV database for feasibility study of Gaia data. We approximated the Gaia sampling of these light curves by the data separated by $\sim 20$ days. We investigated how the variograms were modified by the sampling. An example, the supersoft X-ray source V Sge is displayed in Fig.2. The amount of the sampled data and the length of the mapped time segment roughly correspond to the expected time of observing of Gaia. Since the duration of the high or the low state is longer than the time interval between the sampled data, the profile of the light curve is still recognizable.

Our tests showed that, in the case of the Gaia data, variograms are suitable especially for investigation of the types of CVs which display the light curves, whose profiles are gradual. V Sge with its alternating high and low states and the amplitude of the brightness variations similar for the individual epochs of the cycle is a good example (Fig.3). Similar results can be obtained for novalike CVs with the episodes of the high and low states. However, it is necessary to be cautious in the case of dwarf novae because the outbursts, although with a large amplitude, are usually considerably shorter than the intervals of quiescence. This implies that some outbursts may be missed in the sampled data. It also emerged that binning of the data for variograms (Fig.2) plays a big role. A fine binning yields a more precise determination of the cycle-length, but artifacts and false detections can appear, especially if the data are sampled.

The orbital modulation of CVs can be searched for and investigated in the sampled Gaia data only under some favorable circumstances. Although the length of the orbital period of CV can be considered to be stable (or at most slightly variable) during the proposed lifetime of Gaia, it is reasonable to expect that the orbital modulation will be influenced by the long-term variations (e.g. outbursts, episodes of the high and low states). The amplitude of the long-term variations can often be considerably larger than that of the orbital modulation even in the case of a significant amplitude of this modulation (e.g. $>0.8 \mathrm{mag}$ ). Only if the long-term level of brightness remains almost stable (or if the limited segments of the long-term light curve are selected), the orbital modulation can emerge. A preferable situation will be to fold the Gaia data with the already known orbital period, and to investigate if and how the profile of the modulation varies with the changes between the states of activity. The orbital period can be determined for example by the follow-up observations of the ground-based telescopes. This is one of the cases of co-operation of the orbital and the ground-based observing components of the project.

\section{Conclusions}

Although the two projects covered by this contribution, namely Gaia and LDS photographic surveys, are based on different image recording techniques, and cover different epochs, both of them can effectively contribute to the investigation of CVs.

\section{Acknowledgements}

We acknowledge grant 13-33324S by GACR. We thank Dr I. Savanov for his valuable comments during the referee process. 


\section{References}

[1] Brandt, S., et al., 2013, EAS Publications Series, Volume 61, pp.617-623 (2013)

[2] Davidson, K., et al., ApJ, 220, 239 (1978)

[3] Hudec, R. et al., Acta Polytechnica, Vol. 53, No. 3, p.30 (2013)

[4] Eyer, L. et al., Central European Astrophysical Bulletin, p. 115-126 (2013)

[5] Feroci, M. et al., Experimental Astronomy, Volume 34, Issue 2, pp.415-444 (2012)

[6] Herbig, G. H., et al., ApJ, 141, 617 (1965)

[7] Hudec, L., Algorithms for spectral classification of stars, BSc. Thesis, Charles University, Prague (2007)

[8] Hudec, R., Šimon, V., Specific object studies for cataclysmic variables and related objects ESA Gaia Reference Code GAIA-C7-TN-AIO-RH-001-1 (2007a)

[9] Hudec, R., Šimon, V., Specific object studies for optical counterparts of high energy sources. ESA Gaia Reference Code GAIA-C7-TN-AIO-RH-002-1 (2007b)

[10] Hudec, R., Šimon, V., Hudec, L., Acta Polytechnica, Vol 53, Supplement, p.798 (2013)

[11] Krimm, H. A., et al., ApJS, 209, 14 (2013)

[12] Munari, U., et al., MNRAS, 410, L52 (2011)

[13] Munari, U., et al., MNRAS, 435, 771 (2013)

[14] Šimon, V., Mattei, J. A., A\&AS, 139, 75 (1999)

[15] Steiner, J. E., Diaz, M. P., PASP, 110, 276 (1998)

[16] Szkody. P., et al., AJ, 123, 413 (2002)

[17] van Amerongen, S., van Paradijs, J., A\&A, 219, 195 (1989)

[18] Walker, M., \& Chincarini, G., ApJ, 154, 157 (1968) 